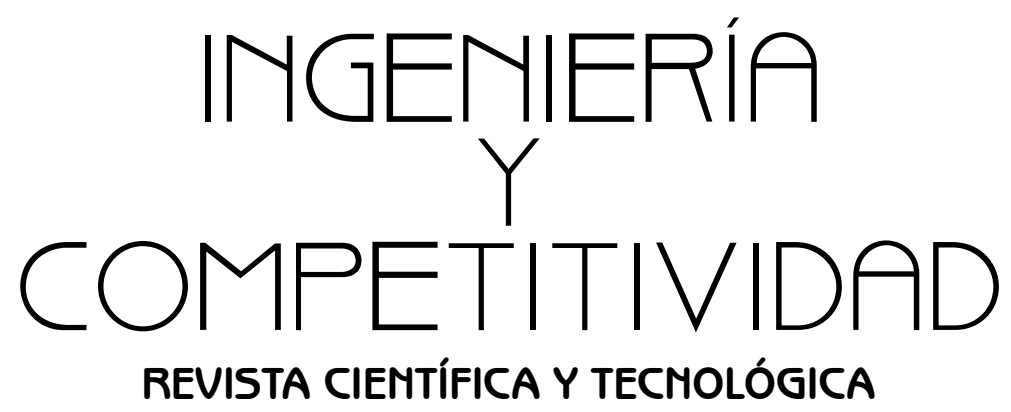

(c) Universidad del Valle - Facultad de Ingeniería

ISSM Mo. 0123 - 3033

Chemical Abstracts Service Abbreviated Title: Ing. Compet.

Volumen 16, Mo. 1, junio de 2014

Institución Editora:

Facultad de Ingeniería - Universidad del Valle

Indexada en:

PUBLINDEX

PERIODICA

Fuente Académica (de EBSCO)

EBSCO INTERMATIOMAL INDEX

CHEMICAL ABSTRACTS

INSPEC

REDALYC

Los conceptos y opiniones de los artículos son responsabilidad exclusiva de su autor; en ningún momento comprometen las orientaciones y políticas de la

Facultad de Ingeniería de la Universidad del Valle.

Los artículos de esta revista pueden descargarse gratuitamente desde la página web http://revistaingenieria.univalle.edu.co y pueden reproducirse libremente citando la fuente.

Comunicaciones de Ingeniería

Teléfonos: (57) (2) 3393195 - Fax: 3302479 - A.A. 25360

Ciudad Universitaria Melendez - Edificio 331 - Torre de Ingeniería - 5piso

e-mail: inycompe@correounivalle.edu.co

http://revistaingenieria.univalle.edu.co

Santiago de Cali, Colombia

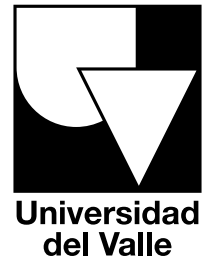

Facultad de Ingeniería 


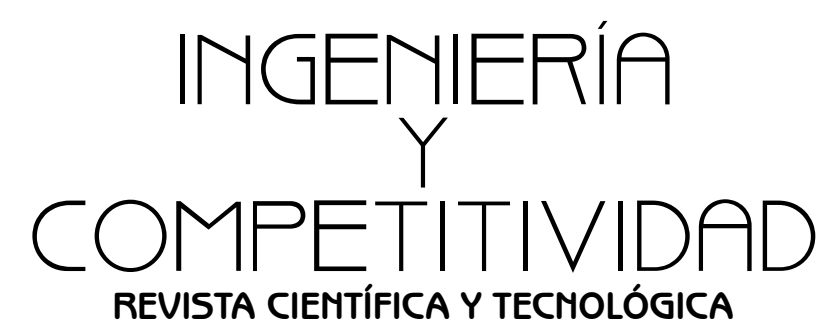

\title{
Dirección universitaria
}

\author{
$\underline{\text { Rector }}$ \\ Iván Enrique Ramos Calderón \\ Vicerrectora de Investigaciones \\ Ángela María Franco Calderón \\ Vicerrector Académico \\ Héctor Cadavid Ramírez \\ Director del Programa Editorial \\ Francisco León Ramírez Potes
}

\section{Facultad de Ingeniería}

\author{
Decano \\ Edgar Quiroga Rubiano \\ Vicedecano de Investigación y Posgrados \\ Patricia Torres Lozada \\ Vicedecano Académico \\ Carlos Rafael Pinedo Jaramillo
}




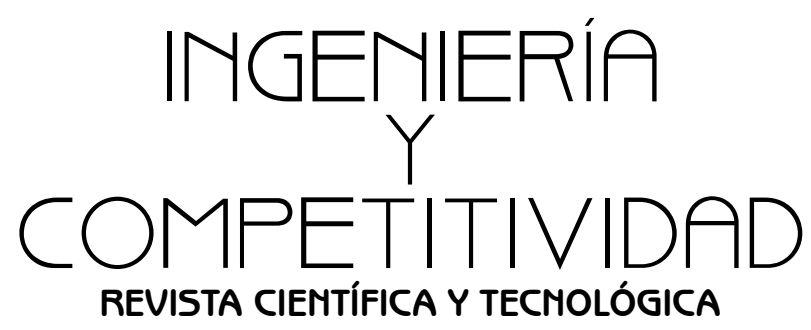

Cuerpo Editorial

Editor Jefe

Fiderman Machuca Martínez, Ph. D.

Joao Luis Ealo Cuello, Ph. D.

Ruby Mejia De Gutierrez, Ph. D.

Miguel Ricardo Peña Varón, Ph. D.

Peter Thomson, Ph.D.

Carlos Julio Vidal Holguín, Ph. D.

Pedro Antonio Moreno Tovar, Ph. D.

Eduardo Behrentz Valencia, Ph. D.

Ramón Alfonso Gallego Rendón, Ph. D.

Carlos Hoffman Sampaio, Ph. D.

Edgar Lotero Alegría, Ph. D.

Jorge Enrique Rodríguez Paez, Ph. D.

Carlos Emilio Romero, Ph. D.

\section{Comité Editorial}

Universidad del Valle (Colombia)

Universidad del Valle (Colombia)

Universidad del Valle (Colombia)

Universidad del Valle (Colombia)

Universidad del Valle (Colombia)

Universidad del Valle (Colombia)

Universidad de los Andes (Colombia)

Universidad Tecnológica de Pereira (Colombia)

Universidad Federal Rio Grande Do Sul (Brasil)

Conoco Phillips (United States of America)

Universidad del Cauca (Colombia)

Lehigh University (United States of America)

\section{Comité Científico}

Jorge Francisco Estela Uribe, Ph. D.

José Luis García Fierro, Ph. D.

Pere Grima Cintas, Ph. D.

Duncan Mara, Ph. D.

Hosahalli Ramaswamy, Ph. D.
Pontificia Universidad Javeriana (Colombia) Instituto de Catálisis y Petroleoquímica (España) Universidad Politécnica de Cataluña (España) University of Leeds (United Kingdom) McGill University (Canada)

Asistente Editorial

Alba Lucía Cifuentes González

Diagramación y Diseño

Edgar Bejarano Soto

Jose R. Regalado 0.

Impresión

Unidad de Artes Gráficas Facultad de Humanidades, Universidad del Valle 